\title{
Effects of Added Silica Nanoparticles on Hectorite Gels
}

\author{
Dzina Kleshchanok,* Vera Meester, Cornelia E. Pompe, Jan Hilhorst, and Henk N. W. Lekkerkerker \\ Van 't Hoff Laboratory, Debye Institute for Nanomaterials Science, Utrecht University, The Netherlands
}

ABSTRACT: We present a study on the macroscopic, microscopic, and rheological behavior of mixtures of natural hectorite clay and different types of anionic Ludox silica spheres. Adding silica spheres to the weak hectorite gels leads the collapse of the suspensions, while the strong gels remain space-filling, though their storage modulus and the yield stress values diminish. We discuss what kind of structural rearrangements are possibly responsible for the macroscopic and rheological changes in the clay/silica mixtures.

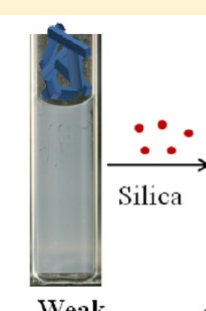

Weak isotropic gel of hectorite clay

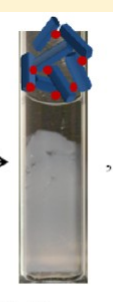

Collapses

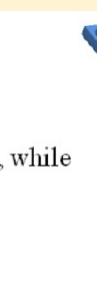$$
\text { b }
$$

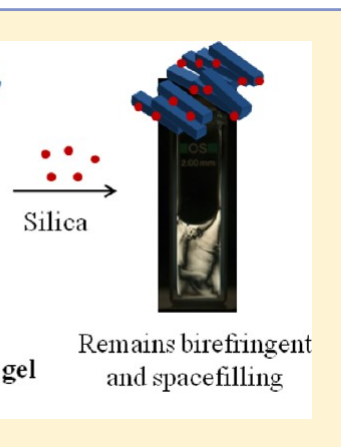

\section{INTRODUCTION}

The addition of minor amounts of small colloids to clay suspensions in a number of cases leads to most interesting, impressive, and even economically important rheological effects.

In a classic paper Schofield and Samson ${ }^{1}$ observed that the addition of small amounts of montmorillonite to kaolinite suspensions profoundly affects the working properties. They argue that the negatively charged montmorillonite particles overlie the positive charges on the edge-faces of the larger kaolinite particles (see Figure 1) leading to deflocculation of the kaolinite suspensions. This manifests itself in the change of the suspension from a stiff paste to a viscous fluid.

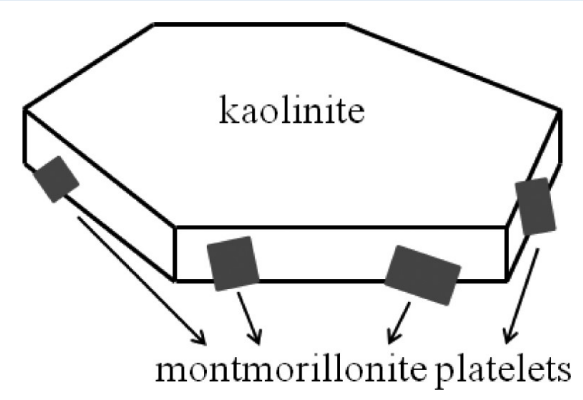

Figure 1. Schematic drawing of the montmorillonite/kaolinite complexes. Redrawn from Schofield and Samson. ${ }^{1}$

The reverse effect was found by Burba and Barnes. ${ }^{2}$ It was observed that upon addition of positively charged mixed metal $(\mathrm{Mg} / \mathrm{Al})$ hydroxide $(\mathrm{MMH})$ particles to a watery slurry of montmorillonite the suspension turned very thick. The unique rheological properties of the montmorillonite/MMH system, such as high yield stress and a severe, reversible drop in viscosity on the application of high shear rates, have found widespread use in the field of drilling and construction fluids (Visplex/Drilplex technology currently in use by M-I drilling fluids). ${ }^{3-5}$ It has been argued that these rheological properties result from the way in which the positive submicrometer-sized
MMH particles complex with the micrometer-sized montmorillonite platelets. In solution the surface of the clay platelets has an excess of negative charge. ${ }^{6} \mathrm{MMH}$ particles align with these negative charges (see Figure 2).,8 Although the attractive forces are individually small, cumulatively they combine to provide a strong gel structure.

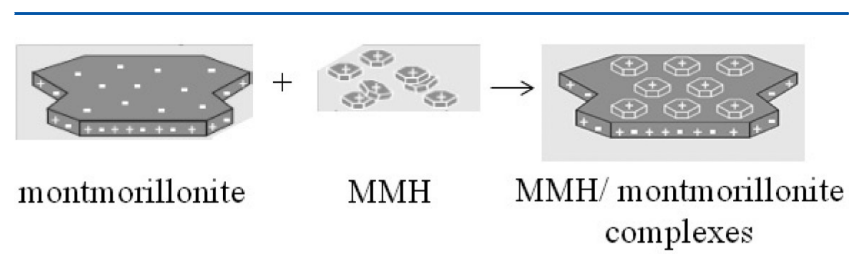

Figure 2. Schematic picture representing the interaction mechanism between montmorillonite clay and $\mathrm{MMH}$ particles. Redrawn from Burba et al. ${ }^{7}$ and Gilmour and Hore. ${ }^{8}$

Van der Kooij et al. ${ }^{9}$ and ten Brinke et al. ${ }^{10}$ discovered that similar rheological behavior could be brought about by the addition of platelike gibbsite and rodlike boehmite colloids to clay suspensions. The advantage of the latter mixed clay-colloid systems is that the effects are very robust and hardly salt dependent, casting doubt as to whether an electrostatic attraction effect is at work.

This leaves open the question whether even simpler and more cost-effective colloidal species could bring about significant rheological effects in clay suspensions. The work of Baird and Walz ${ }^{11,12}$ shows that this is indeed possible. They found that addition of a sufficient amount of anionic silica nanoparticles and salt to aqueous suspensions of kaolinite particles can produce a sol-to-gel transition. The gels exhibit a significant yields stress that increases with the concentration of nanoparticles or salt used in the initial suspension. Ten Brinke et al. ${ }^{10}$ and Bailey et al. ${ }^{13}$ studied the effect of both anionic

Received: March 14, 2012

Revised: June 21, 2012

Published: July 30, 2012 
(Ludox AS, TMA) and cationic (Ludox CL) spherical silica colloids on the rheology of dispersions of well-characterized hectorite and montmorillonite clays, respectively. While the cationic silica particles transform clay dispersions in stiff gels, the action of anionic silica appears to be more ambivalent depending on the initial clay concentration from reducing the viscosity to enhancement of all rheological parameters.

Clearly, the effect of small colloids, in particular silica spheres (a simple, cheap, and commonly available material), on the rheological behavior of clays is rich and varying. To understand and be able to control silica influence on the mechanical properties of clay suspensions is of great practical and economical relevance. In this paper we undertook a systematic scan on hectorite clay suspensions with low added salt amount by studying the mixtures with three different types of anionic silica: Ludox AM, Ludox TMA, and Ludox AS. These three different types of anionic silica not only differ in size but also have subtle but important differences in chemistry. More information can be found from the chart with product description. ${ }^{14}$ We extend the work of ten Brinke et al. ${ }^{10}$ by studying the effect of the variation of the silica concentration and salt content. Depending on the type of Ludox spheres, we find a different rheological behavior of the mixtures. While Ludox AM and TMA always liquefy hectorite gels, the addition of Ludox AS may lead to liquefaction and solidification of the gels depending on the concentrations of clay and spheres. Interestingly, adding silica spheres to the hectorite suspensions, which were in a weak gel state, even leads to a breakup of gel structures.

The paper is organized as follows. After a brief description of samples and instrumentation, we present a study of arrested state formation in aqueous suspensions of pure hectorite clay. Oscillatory rheological measurements were used to locate the appearance of the arrested state region. We show the different states of hectorite suspensions in a phase diagram spanned by the clay concentration and the ionic strength. Next we report optical observations on weak hectorite/silica gels, which collapse with time. Further, the rheological behavior and the confocal microscopy studies of the gelation times of strong hectorite gels with various Ludox silica spheres are reported. We discuss what kind of structural rearrangements can be behind the rheological changes in mixed clay/sphere suspensions. Finally, this paper is summarized with short conclusions.

\section{MATERIALS AND METHODS}

Sample Preparation. Dry hectorite, SHCa-1, was obtained from the Clay Minerals Society, Source Clays Repository. The hectorite suspensions were prepared according to the previously reported procedure. ${ }^{15}$ Hectorite clay belongs to the family of $2: 1$ type smectite clays, with two silicon oxide layers sandwiching a layer of a $\mathrm{Mg}$ oxide. ${ }^{6}$ Hectorite particles are lath-shaped (Figure 3 ). The particles are negatively charged in aqueous solutions due to the isomorphous ion substitution $\left(\mathrm{Mg}^{2+}\right.$ by $\left.\mathrm{Li}^{+}\right)$and the dissociation of silanol groups. However, due to the adsorption of protons, particle edges can also carry a certain amount of positive charges.

Ludox AM, TMA, and AS suspensions were supplied by Sigma-Aldrich and used as received. Particles dimensions were determined from transmission electron microscopy (TEM) images (shown for hectorite in Figure 3) and are presented in Table 1.

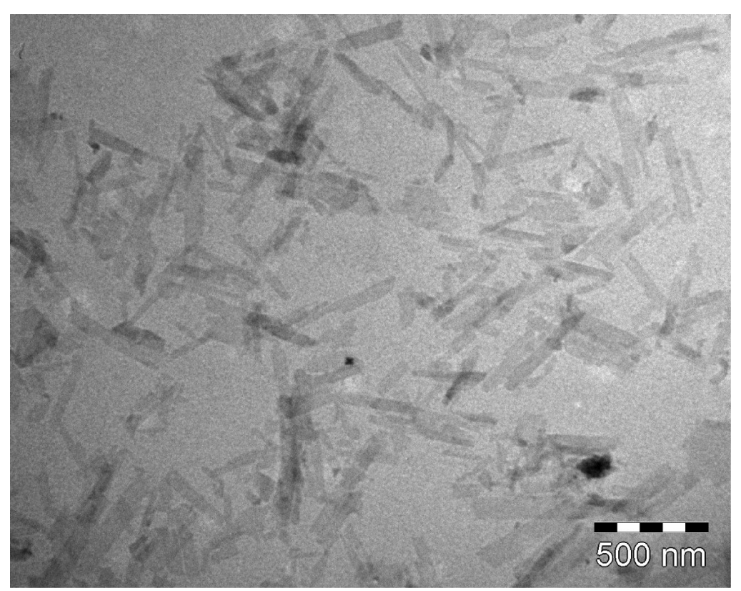

Figure 3. TEM picture of hectorite particles. The scale bar denotes $500 \mathrm{~nm}$.

Table 1. Characteristic Sizes of Hectorite Particles and Silica Spheres Used in This Study

\begin{tabular}{ccccc} 
& hectorite & Ludox AM & Ludox TMA & Ludox AS \\
length, $L / \mathrm{nm}$ & $372.0 \pm 151.0$ & $14.7 \pm 1.6$ & $23.8 \pm 2.5$ & $12.4 \pm 1.8$ \\
width, $W / \mathrm{nm}$ & $50.5 \pm 12.3$ & & & \\
thickness, $^{6}$ & $\sim 1$ & & & \\
$H / \mathrm{nm}$ & & & & \\
\hline
\end{tabular}

Fluorescent hectorite was prepared by dissolving $1.25 \mathrm{mg}$ of rhodamine $\mathrm{B}$ dye in $5 \mathrm{~mL}$ of Millipore water and adding the solution to $120 \mathrm{~g}$ of $1.43 \mathrm{wt} \%$ hectorite dispersion. This mixture was shaken overnight and subsequently centrifuged at $1000 \mathrm{~g}$ for $90 \mathrm{~h}$. Removing the transparent supernatant yielded sediments with a particle concentration of $3.9 \mathrm{wt} \%$.

Mixed clay/sphere samples were prepared by mixing the stock suspensions of hectorite and silica with the solvent (water or various $\mathrm{NaCl}$ solutions) such that the desired colloid and salt concentrations were obtained. All samples were vigorously stirred before further investigations took place.

Rheology. Measurements were performed on a stresscontrolled rheometer (Physica MCR-300) in a sandblasted cone $\left(25 \mathrm{~mm}, 1^{\circ}\right)$ and plate geometry. A solvent trap (Anton Paar) was used to minimize sample evaporation. We performed oscillatory stress sweep measurements at frequency, $f$, of $1 \mathrm{~Hz}$ to find a linear viscoelastic regime (LVE) for all samples. Frequency sweeps were performed then in the LVE regime. The $f$ was decreased from 10 to $0.01 \mathrm{~Hz}$. Flow curves were obtained in controlled ramp up shear stress, $\tau$, tests. Before each measurement the samples were presheared at a high strain amplitude (1000\%) and a $f$ of $1 \mathrm{~Hz}$ for $5 \mathrm{~min}$ to avoid effects from loading and shear history. Afterward a waiting time of 5 min was given to the samples to regenerate their structure.

Optical Observations. Mixtures for visual observations were put into the glass capillaries $(1.0 \times 10.0 \mathrm{~mm}$ cross section, Vitrotubes, VitroCom Inc.) that were flame-sealed. The samples were stored vertically at $20{ }^{\circ} \mathrm{C}$, and changes in their macroscopic appearance were pictured using a Nikon D80 camera with a $50 \mathrm{~mm}$ f1.8 lens. Birefringence of the samples was checked with crossed polarizers and when found present indicated an orientationally ordered structure of the sample.

Confocal Microscopy. For confocal microscopy, a Nikon Eclipse TE2000U inverted microscopy with a Nikon C1 confocal scan head was used. A HeNe (543.5 nm) laser was used for illumination, and detection was done with a 
photomultiplier tube. Sample cells were made of Chromacol 2SV chromatography vials with sawed-off bottoms. The bottoms were replaced by gluing microscope coverslips to the vials using Araldit AW2101 epoxy glue with HW2951 hardener. Samples were shaken vigorously for $5 \mathrm{~min}$ and imaged immediately afterward.

Microscope Autocorrelation Functions. To quantify the effects observed in confocal images, intensity autocorrelation functions were calculated according to

$$
g=\frac{\left\langle I\left(t_{0}\right) I\left(t_{0}+\Delta t\right)\right\rangle_{\mathrm{p}}}{\left\langle I\left(t_{0}\right)\right\rangle_{\mathrm{p}}\left\langle I\left(t_{0}+\Delta t\right)\right\rangle_{\mathrm{p}}}
$$

where $g$ represents the correlation function, $I\left(t_{0}\right)$ is the intensity of a single image pixel at a time $t_{0}$, and $I\left(t_{0}+\Delta t\right)$ is the intensity of the same pixel at a time $\Delta t$ later. \langle\rangle$_{\mathrm{p}}$ represents averaging over all pixels. Normalized to $\left\langle I\left(t_{0}\right)^{2}\right\rangle_{\mathrm{p}}$, such correlation functions have values between 1 (fully correlated, exact same images) and 0 (fully uncorrelated).

\section{EXPERIMENTAL RESULTS}

Pure Hectorite Suspensions. State and Phase Diagram of Hectorite Clay. We used oscillatory rheological measurements to determine an arrested state in hectorite suspensions. Frequency sweeps were performed in the LVE regime and storage, $G^{\prime}$, and loss, $G^{\prime \prime}$, moduli were measured. An arrested state was identified in suspensions where $G^{\prime}$ was larger than $G^{\prime \prime}$ and both moduli were $f$ independent and larger than $1 \mathrm{~Pa}$. Figure 4 presents frequency sweeps for a series of hectorite concentrations in a $10^{-4} \mathrm{M} \mathrm{NaCl}$ solution. The suspensions with clay concentrations equal to and above $0.5 \mathrm{wt} \%$ are arrested. The $G^{\prime}$ modulus rises with an increasing hectorite concentration and reaches a large value of $200 \mathrm{~Pa}$ in a $2.0 \mathrm{wt} \%$ suspension.

Combining the oscillatory measurements on the samples with different clay and salt concentrations, we draw a phase and state diagram of pure hectorite suspensions (Figure 5). A striking feature of this diagram is that the suspensions are arrested already at $0.5 \mathrm{wt} \%$ at all salt concentrations studied. This value is several times lower than for laponite clay (a wellstudied synthetic hectorite). ${ }^{16}$

Additional observations of the arrested samples between crossed polarizers revealed that the suspensions above $1.5 \mathrm{wt} \%$ are birefringent (Figure 5). We denote an ordered structure of these samples as nematic with hectorite particles oriented along a common director. The birefringent hectorite suspensions all have a large $G^{\prime}(>70 \mathrm{~Pa})$, being strong gels. We call these gels nematic gels (NG) as in works of Mourchid et al. ${ }^{17}$ and Gabriel et al. ${ }^{18}$

On the other hand, arrested suspensions below $1.5 \mathrm{wt} \%$ are nonbirefringent (Figure 5). They are isotropic gels (IG) with a disordered structure. A possible example could be a house-ofcards structure, ${ }^{6}$ which is built due to an electrostatic attraction between oppositely charged faces and edges of clay particles. Due to the small surface area of the edges, such a structure would lead to weaker gels, having lower $G^{\prime}$. Indeed, the nonbirefringent suspensions have a $G^{\prime}$ lower than $30 \mathrm{~Pa}$.

We are aware of the ongoing discussion on the nature of the arrested states in clay suspensions, such as gel or glass. ${ }^{16,19-21}$ However, despite differences in the $G^{\prime}$ values and the ability to polarize light, we cannot assign the observed arrested states of hectorite to different classes. Thus, we denote them as a weak (isotropic, IG) and a strong (nematic, NG) gel. The transition

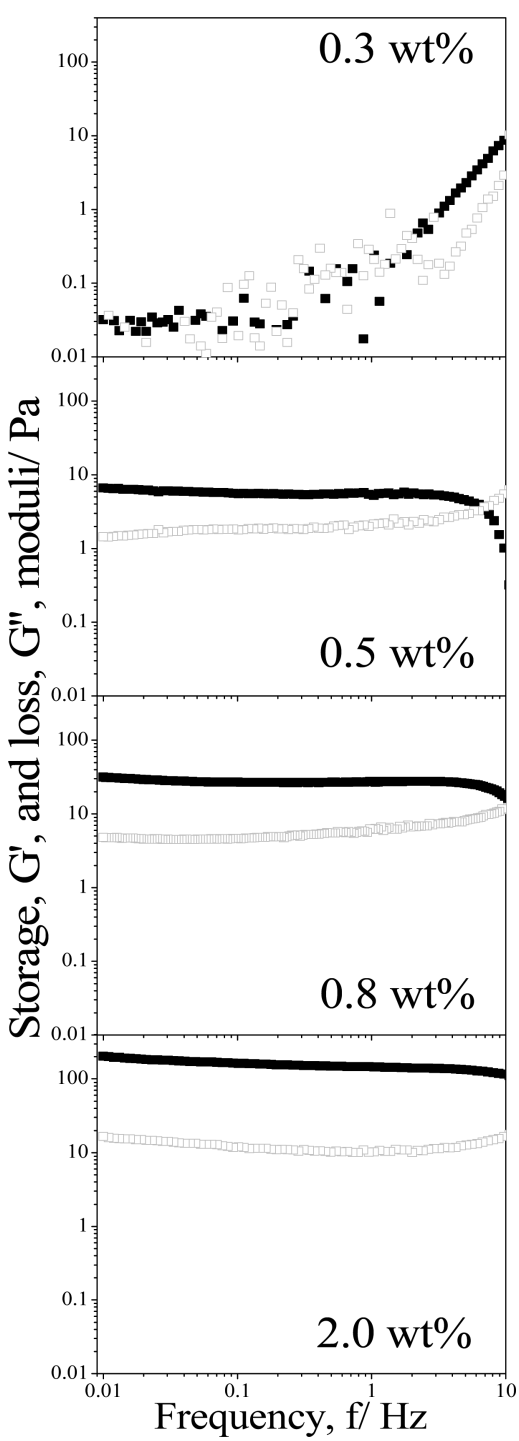

Figure 4. Storage (black squares) and loss (gray squares) moduli vs $f$ of pure hectorite suspensions in a $10^{-4} \mathrm{M} \mathrm{NaCl}$ solution. The weight concentrations of hectorite, stated in the graph, are increasing from top to bottom.

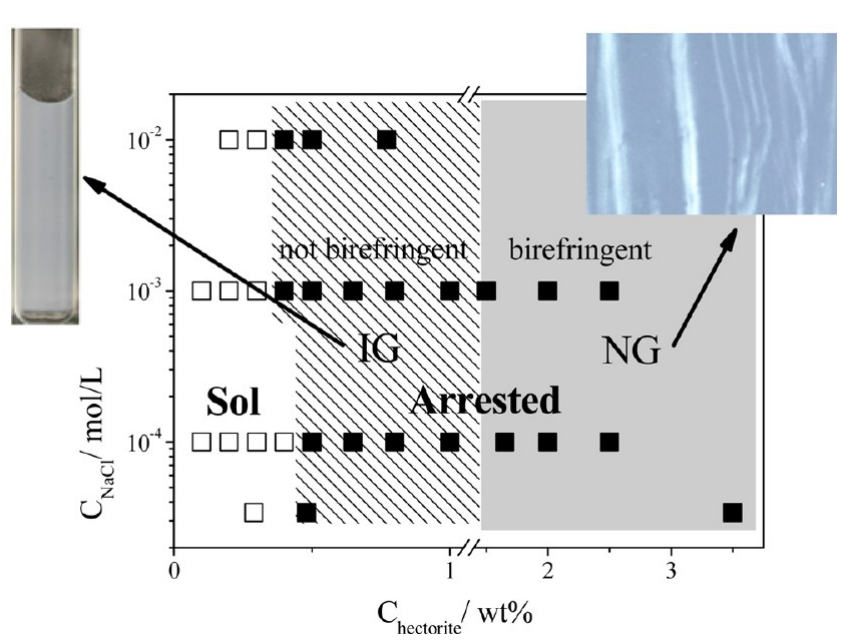

Figure 5. Phase and state diagram of pure hectorite suspensions. Arrested states are weak isotropic (IG) and strong nematic (NG) gels. 
a)
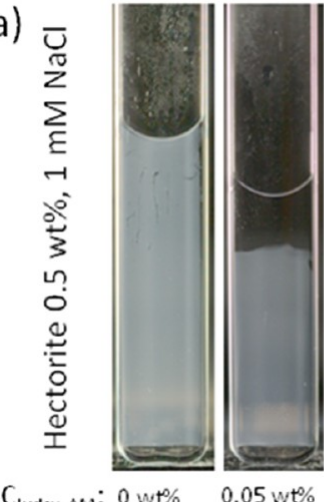
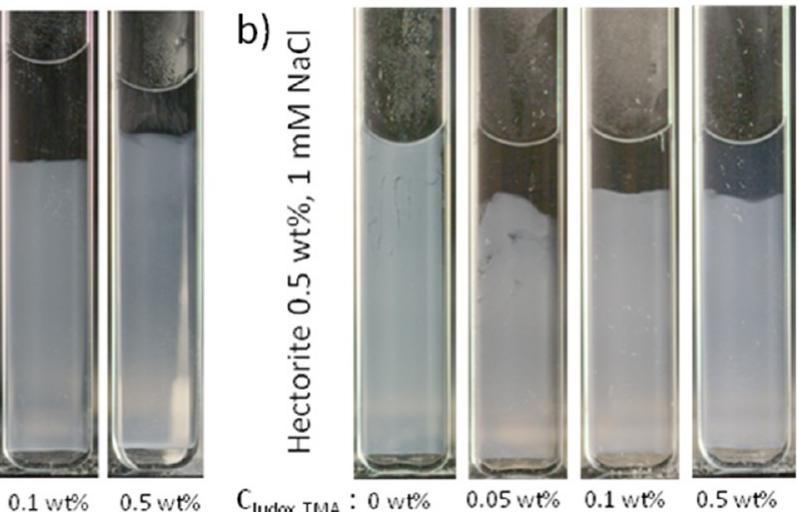

Figure 6. Macroscopic appearance of (a) pure hectorite $(0.5 \mathrm{wt} \%)$ and hectorite/Ludox AM samples and (b) pure hectorite (0.5 wt \%) and hectorite/Ludox TMA samples. While the hectorite suspension is a space-filling gel, the mixtures show two phases.
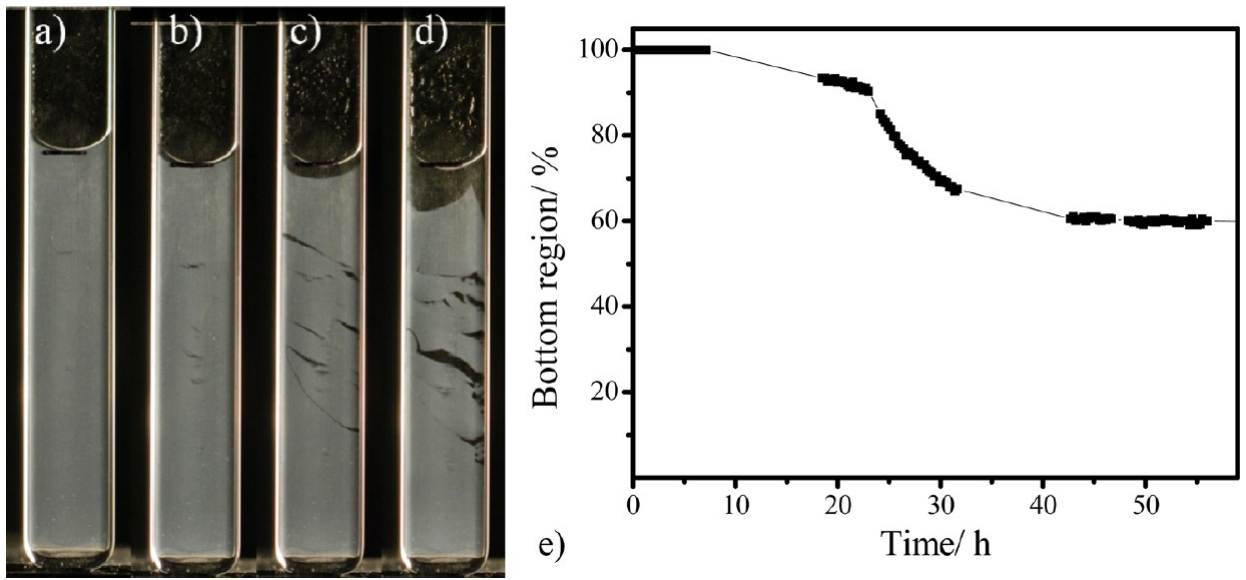

Figure 7. Summary of the collapse of the hectorite $(0.5 \mathrm{wt} \%$ in $1 \mathrm{mM} \mathrm{NaCl}) /$ Ludox AM $(0.1 \mathrm{wt} \%)$ sample: (a) initial suspension, (b) appearance, and (c) growth of cracks, (d) collapsed sample, (e) amount of the turbid bottom phase vs time (the line is a guide for the eyes).

between the two of them agrees well with the overlap concentration $c^{*}{ }^{15}$

Hectorite Plus Silica Nanospheres. Collapse of Weak Hectorite/Silica Gels. When silica spheres are added to the suspensions of hectorite, which are in a weak gel state (located on the left border of the arrested state region, see Figure 5), a transition is seen from a space-filling gel to a sample with two regions. Figure 6 shows macroscopic pictures of a pure hectorite sample $(0.5 \mathrm{wt} \%$ in $1 \mathrm{mM} \mathrm{NaCl})$ and the mixtures of hectorite and Ludox AM (Figure 6a) and Ludox TMA (Figure $6 \mathrm{~b}$ ) (concentration varied from 0.05 to $0.5 \mathrm{wt} \%$ ). The mixed samples consist of a bottom turbid and a transparent upper region. The TEM analysis of the upper phase showed that there are virtually no colloids in it; neither clay nor Ludox particles are present there. This finding is different from the one on mixtures of gibbsite platelets and silica Ludox CL spheres, ${ }^{22}$ where a partitioning of colloids took place, with most of the spheres located in the upper and most of the platelets in the bottom phase. While in gibbsite/Ludox CL mixtures depletion attraction ${ }^{23}$ is responsible for the formation of two phases, it is not the case for hectorite/Ludox AM and TMA samples. It is because of the small amount of the depletant (silica spheres), which only could lead to a depletion attraction of $\max 1 k_{\mathrm{B}} T$, not sufficient to cause a phase transition. Second evidence is the absence of a depletion agent in the upper phase.

The transition from a space-filling gel to a two-region sample in the hectorite/silica mixtures always follows a certain scenario.
Figure 7 illustrates an example of hectorite/Ludox AM spheres. At first, for a long time no changes happen in the system (Figure 7a). Then small cracks start to appear (Figure 7b) and grow significantly in size (Figure 7c), which leads to a sudden collapse of the gel (Figure $7 \mathrm{~d}$ ). Thus, the process consists of an induction time $(20 \mathrm{~h})$, a sudden collapse of a gel (at 24th hour), and a slow compression of the bottom phase (until the 40th hour), after which no changes happen in the system (Figure 7e).

Rheological Measurements. Figure 8 presents frequency sweeps on mixed hectorite/Ludox AM, hectorite/Ludox TMA, and hectorite/Ludox AS suspensions. The $G^{\prime \prime}$ modulus was always lower than $G^{\prime}$ and is not shown in the figure for clarity reasons. The hectorite suspensions were strong gels with clay concentrations of 2.5 wt \% (Figure 8a, b, and c) and 3.5 wt \% (Figure $8 \mathrm{~d}, \mathrm{e}$, and $\mathrm{f}$ ). The concentration of spheres varied from 0.03 to $2.0 \mathrm{wt} \%$. The plots reveal a similar influence of Ludox AM and TMA spheres on the rheological behavior of hectorite suspensions. Samples with both hectorite concentrations become more liquidlike with addition of the spheres. The $G^{\prime}$ modulus significantly drops, and the decrease is generally more pronounced with an increasing concentration of spheres. The effect of Ludox AS is not so pronounced and unambiguous. The $G^{\prime}$ modulus of mixed hectorite/Ludox AS suspensions is smaller but stays close to the $G^{\prime}$ modulus of pure hectorite samples. Interestingly, the hectorite $(2.5$ wt \%) mixture with 0.2 wt \% of Ludox AS has a $G^{\prime}$ value that is $70 \mathrm{~Pa}$ higher than the 

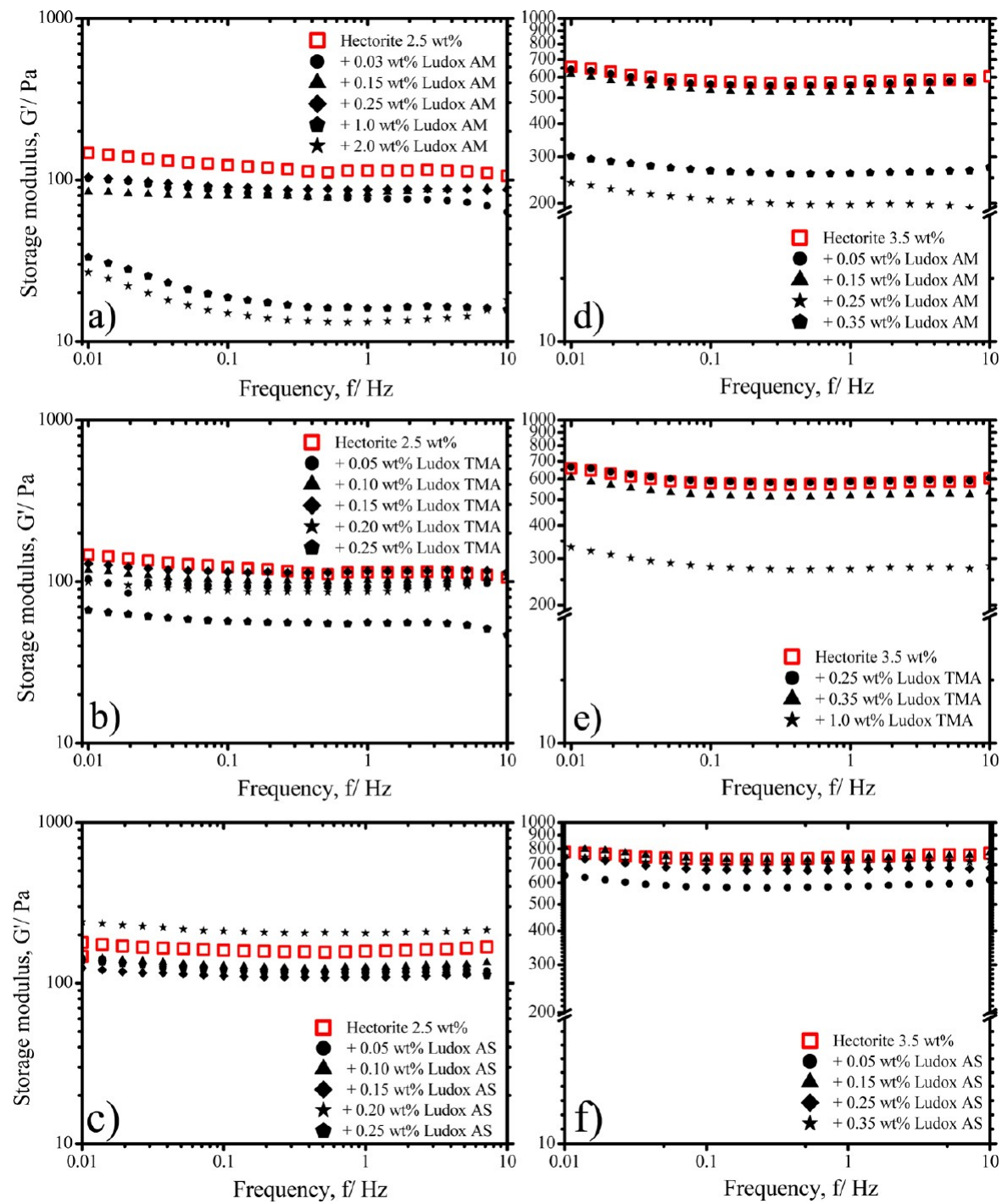

Figure 8. Frequency sweeps from mixed suspensions: (a) hectorite (2.5 wt \%)/Ludox AM, (b) hectorite (2.5 wt \%)/Ludox TMA, (c) hectorite (2.5 wt \%)/Ludox AS, (d) hectorite (3.5 wt \%)/Ludox AM, (e) hectorite (3.5 wt \%)/Ludox TMA, and (f) hectorite (3.5 wt \%)/Ludox AS. Storage moduli from pure hectorite suspensions are shown as hollow red squares.

hectorite suspension. However, all other concentrations of Ludox AS added to the clay lowered the $G^{\prime}$ value.

Figure 9 shows the flow curves of the aforementioned suspensions in a $\tau$ vs shear rate, $\dot{\gamma}$, representation on a doublelogarithmic scale. We see that pure hectorite suspensions have yield stress, the critical stress above which the material starts to flow, ${ }^{24}$ as manifested by the horizontal jump in shear rate at constant stress in the center of the flow curves. With "yield stress" we mean an "effective, if not real yield stress" at which failure of the gel occurs. While the $2.5 \mathrm{wt} \%$ hectorite suspension has a yield stress of $20 \mathrm{~Pa}$, its value increases to 50 $\mathrm{Pa}$ for 3.5 wt \% suspension. The addition of Ludox AM or TMA spheres lowers the yield stress. Suspensions with the largest amount of spheres ( 1 and 2 wt \% of Ludox AM) have a reduced yield stress. Again, suspensions with Ludox AS have flow curves very similar to the pure hectorite samples, and the mixture hectorite $(2.5 \mathrm{wt} \%) /$ Ludox AS (0.2 wt \%) has slightly larger yield stress.

Confocal Microscopy. To supplement the data obtained with rheology and to gain information on the processes occurring on a local time and structure scale, confocal microscopy was performed on dispersions of dyed hectorite. Using this technique, 3D information on sample structures could be obtained up to $50 \mu \mathrm{m}$ deep into a sample. From visual inspection, a clear difference was apparent between samples of strong hectorite gels $(2.5 \mathrm{wt} \%)$ and similar samples with a high concentration of added Ludox AS (1.0 wt \%). Confocal micrographs taken at $5 \mu \mathrm{m}$ from the sample wall are shown in Figure 10. Figures $10 \mathrm{a}-\mathrm{c}$ show confocal microscopy images of a sample containing $2.5 \mathrm{wt} \%$ hectorite clay without added silica at different times after rejuvenation. Close investigation (follow white arrows in Figures $10 \mathrm{a}-\mathrm{c}$ ) shows that the images taken at different times are nearly identical, indicating a rigid structure and therefore a quick gelation after rejuvenation. Figures $10 \mathrm{e}-\mathrm{g}$ show micrographs of a sample containing the same concentration of hectorite and an additional $1.0 \mathrm{wt} \%$ of silica Ludox AS spheres. Between images 10e and 10f, taken directly after and $5 \mathrm{~min}$ after rejuvenation, respectively, many differences can be observed. Only a few features remain stationary (indicated by the top left and bottom left white arrows), while other features change. Between images $10 \mathrm{f}$ and 

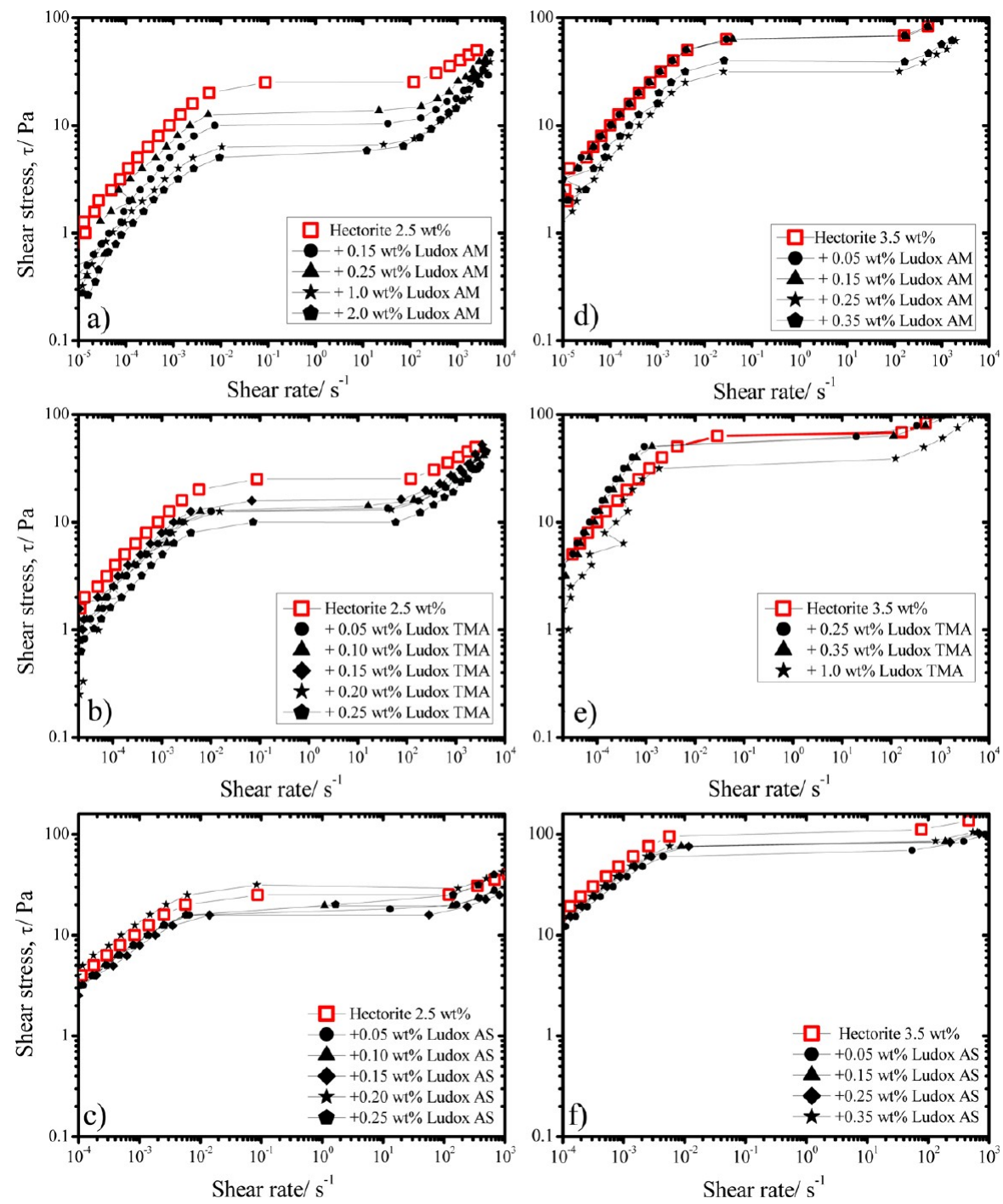

Figure 9. Flow curves obtained in a controlled shear stress regime on mixed suspensions: (a) hectorite (2.5 wt \%)/Ludox AM, (b) hectorite (2.5 wt \%)/Ludox TMA, (c) hectorite (2.5 wt \%)/Ludox AS, (d) hectorite (3.5 wt \%)/Ludox AM, (e) hectorite (3.5 wt \%)/Ludox TMA, and (f) hectorite $(3.5 \mathrm{wt} \%) /$ Ludox AS. Flow curves of pure hectorite suspensions are shown as hollow red squares.

$10 \mathrm{~g}$, all movement has slowed down and the difference between these images is not as pronounced as between $10 \mathrm{e}$ and $10 \mathrm{f}$.

To quantify the difference between the samples, intensity autocorrelation functions were calculated as described in the experimental section. The curves in Figure 10d hardly decay until several tens of seconds after the reference time. This indicates that at such time scales, the recorded images are almost identical and samples are essentially immobile. The pure hectorite suspension is able to rebuild its structure very fast after rejuvenation. Looking at the curves in Figure 10h, the difference from the hectorite/Ludox AS mixture is immediately apparent. At a time difference of $10 \mathrm{~s}$, which is the shortest time scale available in our experiments, a significant loss in correlations is already present. If $t_{0}$ (the time at which the reference image was taken) is chosen to be immediately after rejuvenation, the initial decay is strongest. The magnitude of the initial decay gradually decreases when $t_{0}$ increases, signifying a slow gelation of the sample. At $t_{0}=500 \mathrm{~s}$, the decay is almost absent and the sample is almost fully gelled, yet still weaker than the sample without silica, as also observed in rheological measurements.
This example shows the potential for using microscopy to supplement rheological data. A single confocal data set can qualitatively reproduce trends that were also observed in rheology, but with a better time resolution and on a local scale. In addition, confocal microscopy is noninvasive, so the measurements minimally affect the gel structure. For future measurements on truly complex gelled systems, a combination of rheology with confocal microscopy will make a powerful toolbox with the potential to correlate local changes with bulk behavior.

\section{DISCUSSION}

To summarize the experimental findings, hectorite clay suspensions form an arrested state already at a very low concentration $(0.5 \mathrm{wt} \%)$ at all ionic strengths studied (up to 10 $\mathrm{mM} \mathrm{NaCl}$ ). This concentration value is several times lower than that of a synthetic hectorite, laponite, due to the fact that natural hectorite particles are more anisotropic. The lengthoverthickness, $L / H$, and the width-overthickness, $W / H$, values of hectorite are significantly larger than of laponite, which is important for a sol/gel transition. ${ }^{25}$ 

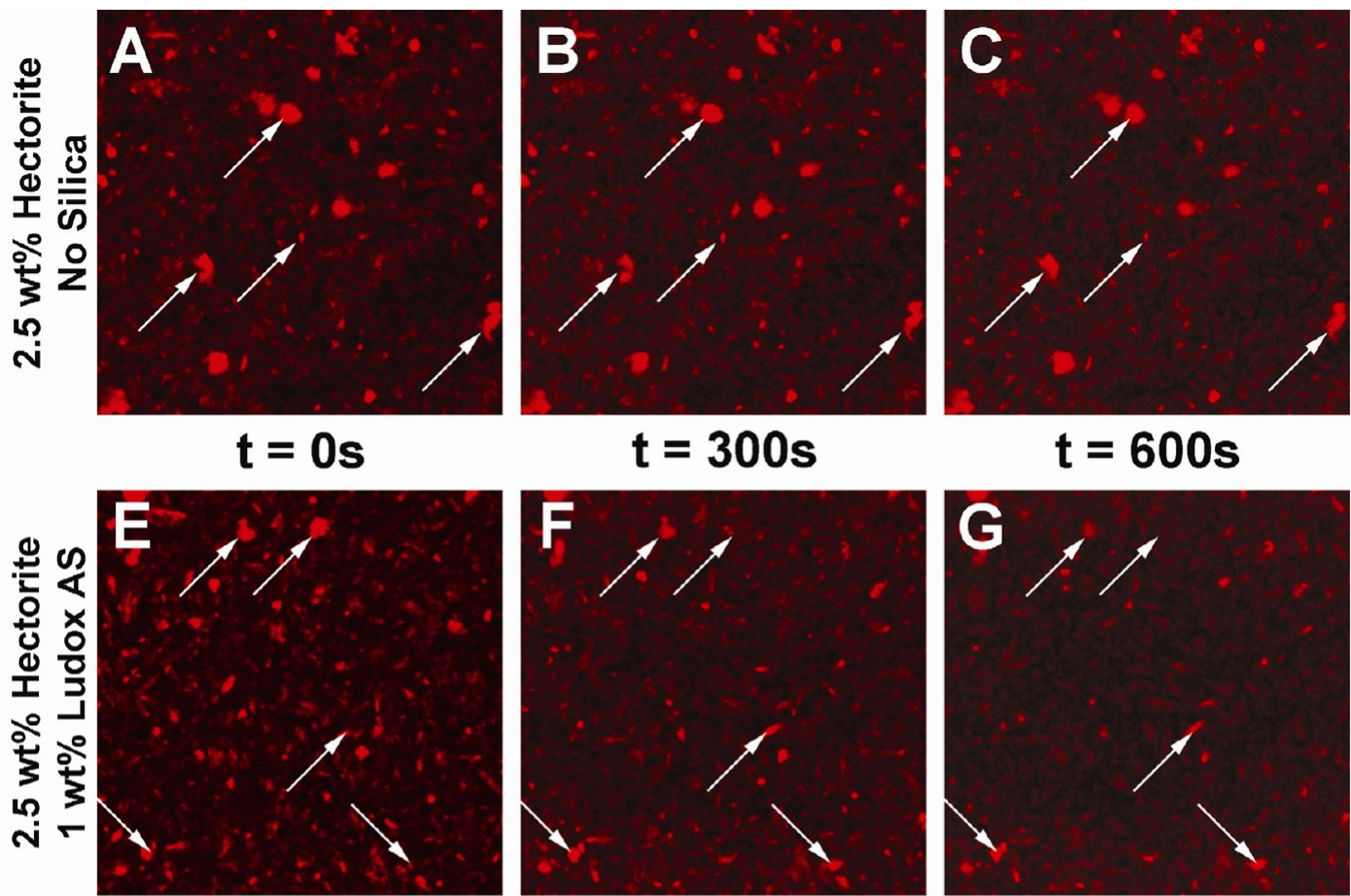

$t=300 s$

$t=600 s$
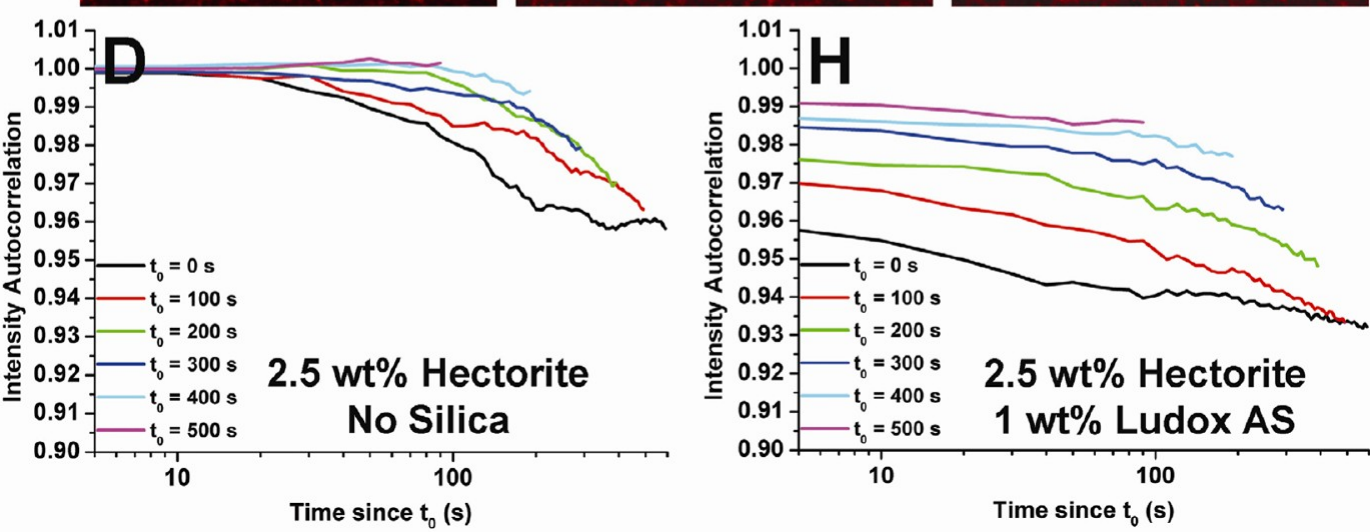

Figure 10. (A-C) Confocal images of pure, $2.5 \mathrm{wt} \%$ hectorite dispersion at various times after homogenization. (D) Intensity autocorrelation functions for the same hectorite dispersion with various $t_{0}$-values. $(E-G)$ Confocal images of a 2.5 wt \% hectorite dispersion with 1.0 wt $\%$ of Ludox AS at various times after homogenization. $(\mathrm{H})$ intensity autocorrelation functions for the same dispersion with various $t_{0}$-values. White arrows are guides to follow the static or changed structure of the gels.

When adding spherical silica colloids to the strong hectorite gels, in almost all cases a liquefaction of the suspensions takes place. Both the $G^{\prime}$ and the yield stress are lower in the mixed samples than in the pure clay suspensions. Surprisingly, the decrease of these values is not always proportional to the sphere concentration in the systems. We observe significant differences in the action of the type of Ludox added. This might be related to the size, charge, and accompanying counterions of the different Ludox species. ${ }^{14}$ This clearly requires further exploration at the particle/interactions level.

Interestingly, mixed suspensions with lower clay contents (weak isotropic gels) become so liquidlike that they cannot sustain their weight and collapse. The collapse is sudden and appears after a prolong induction time. This behavior is similar to the delayed sedimentation of transient polymer/colloid gels. ${ }^{26}$ However, in those gels the force responsible for the macroscopic changes was a weak depletion attraction due to the nonadsorbing polymer chains. An estimated value ${ }^{22}$ of the depletion attraction at the highest silica amount in the clay mixtures is only $1 k_{\mathrm{B}} T$, which is not enough to bring a system to an arrest or a phase transition.

Following the line of reasoning of Schofield and Samson, ${ }^{1}$ we presume that the opposite charges of hectorite edges (positive) and silica spheres (negative) are responsible for the changes in the clay microstructure. Figure 11 schematically presents what kind of microstructural rearrangements are possibly responsible for the liquefaction of the hectorite/silica mixtures. Spherical colloids can absorb on the clay edges. This would prevent a house-of-card configuration of the weak gels (Figure 11a). ${ }^{6}$ Thus, the structure of the mixtures becomes significantly more loose and disentangled and eventually collapses (Figure 11a). The addition of silica spheres to the strong gels leaves the birefringence in place (Figure 11b). Nevertheless, the rheological properties are diminished. In the case of the strong gels, clay faces would be located further apart from each other due to the adsorption of the spheres. This would lead to the weakening of the interactions between the clay particles. 
a)

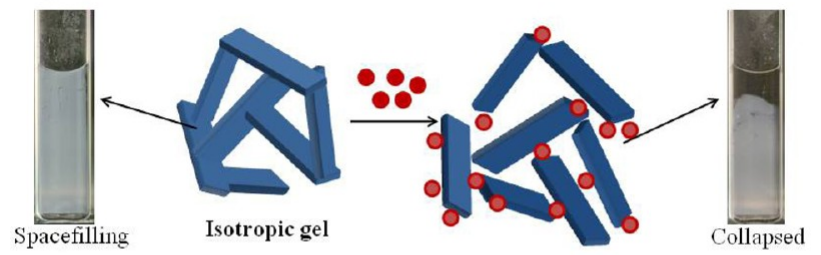

b)

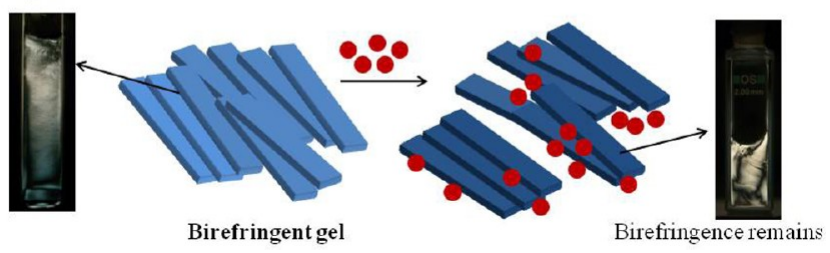

Figure 11. Schematic representation of the microstructural changes responsible for the liquefaction of (a) a weak isotropic and (b) a strong nematic hectorite gel with the addition of silica spheres.

\section{CONCLUSIONS}

We present a systematic study of the phase and state behavior of the pure hectorite suspensions. Oscillatory rheological measurements were applied to locate the appearance of the sol and arrested states. We summarize our findings in the phase diagram spanned by the clay concentration and the ionic strength. Further, various types of Ludox spheres (AM, TMA, AS) were used in mixtures with hectorite, and their influence on the rheological properties of the clay suspensions was studied. Confocal microscopy studies show that hectorite/silica gels are very different from the pure hectorite gels in the ability to rebuild the gel structure after rejuvenation. On a base of our experimental observations, we speculate that structural rearrangements, due to the adsorption of negatively charged spheres to the positively charged clay edges, bring the changes to the macroscopic and rheological behavior of hectorite clay suspensions.

\section{AUTHOR INFORMATION}

\section{Corresponding Author}

*E-mail: D.Kleshchanok@uu.nl.

\section{Notes}

The authors declare no competing financial interest.

\section{ACKNOWLEDGMENTS}

Louise Bailey and Geoff Maitland are kindly acknowledged for many insightful discussions. This work is part of the research program of the Foundation for Fundamental Research on Matter (FOM), which is part of The Netherlands Organization for Scientific Research (NWO).

\section{REFERENCES}

(1) Schofield, R. K.; Samson, H. R. Discuss. Faraday Soc. 1954, 135.

(2) Burba, J. L., III; Barnes, A. L. Mixed Metal Layered HydroxideClay Adducts As Thickeners for Water and Other Hydrophylic Fluids. U.S. Patent 4790954, May 7, 1987

(3) Fraser, L. J.; Reid, P.; Williamson, D.; Enriquez, F. SPE Annual Technical Conference, 1995; pp SPE 30501.

(4) Fraser, L. J.; Reid, P.; Williamson, D.; Enriquez, F. SPE Drill. Completion 1999, 14, 178.

(5) Fraser, L. J.; Aragao, A. F. L. SPE/IADC Drilling Conference, 2001; pp SPE/IADC 67734.
(6) van Olpen, H. An Introduction to Clay Colloid Chemistry; Interscience: New York, 1963.

(7) Burba, J. L., III; Holman, W. E.; Crabb, C. R. IADC/SPE Drilling Conference, 1988.

(8) Gilmour, A.; Hore, N. AADE Annual Technical Forum, 1999.

(9) van der Kooij, F. M.; Lekkerkerker, H. N. W.; Boek, E. S. Process Fluid. Pat. Appl. 0200350, 2008

(10) ten Brinke, A. J. W.; Bailey, L.; Lekkerkerker, H. N. W.; Maitland, G. C. Soft Matter 2008, 4, 337.

(11) Baird, J. C.; Walz, J. Y. J. Colloid Interface Sci. 2006, 297, 161.

(12) Baird, J. C.; Walz, J. Y. J. Colloid Interface Sci. 2007, 306, 411.

(13) Bailey, L.; Lekkerkerker, H. N. W.; Maitland, G. C. To be published.

(14) Ludox-Technical Literature; E. I. du Pont de Nemours and Company: Wilmington, DE, 1999.

(15) ten Brinke, A. J. W.; Bailey, L.; Lekkerkerker, H. N. W.; Maitland, G. C. Soft Matter 2007, 3, 1145.

(16) Ruzicka, B.; Zaccarelli, E. Soft Matter 2011, 7, 1268.

(17) Mourchid, A.; Lecolier, E.; van Damme, H.; Levitz, P. Langmuir 1998, 14, 4718 .

(18) Gabriel, J. C. P.; Sanchez, C.; Davidson, P. J. Phys. Chem. 1996, $100,11139$.

(19) Ruzicka, B.; Zulian, L.; Zaccarelli, E.; Angelini, R.; Sztucki, M.; Moussaid, A.; Ruocco, G. Phys. Rev. Lett. 2010, 104, 085701.

(20) Mongondry, P.; Tassin, J. F.; Nicolai, T. J. Colloid Interface Sci. 2005, 283, 397.

(21) Tanaka, H.; Meunier, J.; Bonn, D. Phys. Rev. E 2004, 69, 031404.

(22) Kleshchanok, D.; Petukhov, A. V.; Holmqvist, P.; Byelov, D. V.; Lekkerkerker, H. N. W. Langmuir 2010, 26, 13614.

(23) Lekkerkerker, H. N. W.; Tuinier, R. Colloids and the Depletion Interaction; Springer: Dordrecht, Heidelberg, London, New York, 2011.

(24) Mewis, J.; Wagner, N. J. Colloidal Suspension Rheology; Cambridge University Press: Cambridge, U.K., 2012.

(25) Solomon, M. J.; Spicer, P. T. Soft Matter 2010, 6, 1391.

(26) Poon, W. C. K.; Starrs, L.; Meeker, S. P.; Moussaid, A.; Evans, R. M. L.; Pusey, P. N.; Robins, M. M. Faraday Discuss. 1999, 112, 143. 